\title{
A Quick Generation Method of Sequence Pair for Block Placement
}

\author{
Mingxu Huo and Koubao Ding \\ Dept. of Information and Electronic Engineering, Zhejiang University, \\ Hangzhou 310027, P.R. China \\ $\{$ huomingxu, dingkb\}@zju.edu.cn
}

\begin{abstract}
Sequence Pair (SP) is an elegant representation for the block placement of IC Design, and it is usually imperative to generate the SP from an existing placement. A quick generation method and one concise algorithm are proposed instead of the original unfeasible one. It is also shown that if the relations of any two blocks are either vertical or horizontal, the solution space size of a representation is $(\mathrm{n} !)^{2}$ if it is $\mathrm{P}^{*}$-admissible. The analytical and experimental results of the algorithm both show its superiority in running time.
\end{abstract}

\section{Introduction}

With the rapid increase of IC complexity, floorplanning and block placement become more important, where floorplanning can be regarded as placement with soft module blocks. Most of such complex combinatorial optimization problems are NP-Hard [1], and therefore heuristic approaches such as Simulated Annealing algorithms are widely used to generate good layouts. The representation of geometrical topological relations of blocks is one of the crucial factors in evaluating values of the cost functions. Without some additional procedures, most representations proposed in the literature usually cannot include optimal solution, while Sequence Pair (SP) [1], BSG [2], TCG [3] and TCG-S [4] etc are $\mathrm{P}^{*}$-admissible representations $[1,4]$ that can represent the most general floorplans and contain a complete structure for searching an optimal solution. The original method to generate SP is named Gridding [1], which is too complicated to be implemented. However, it is imperative to find an effective and efficient approach to generate SP from arbitrary existing placement.

\section{Generation Method}

The topological relations of any two non-overlap module blocks are horizontal and vertical, i.e., left to, right to, above and below [5]. Diagonal relations also exist, but they can be simply degenerated by preferring horizontal relations to vertical ones, or by adopting the relation definitions in [3]. An HV-Relation-Set (HVRS) for a set of blocks is a set of horizontal or vertical relations for all block pairs [6], and a FeasibleHVRS involves all the block relations excluding the non-realizable ones.

A Sequence Pair (SP) is an ordered pair of sequences $\Gamma_{+}$and $\Gamma_{-}$, each of which is a sequence of $n$ block names [1], e.g., in Fig. 1 is one of the possible placements of the 
apte circuit from MCNC benchmark ${ }^{1}$ circuits, the SP from which is $\left(\Gamma_{+}, \Gamma_{-}\right)=$ $(670185423,120634785)$. A sequence pair corresponds to a Feasible-HVRS.

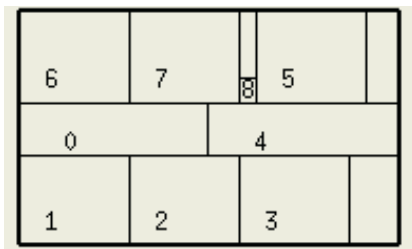

Fig. 1. A placement of the apte MCNC benchmark circuit. Its SP is $(670185423,120634785)$

A generation plane is an infinite plane with numerous different positions. All blocks are treated as symbol points, each of which will be embedded in one unique position in the generation plane according to the Feasible-HVRS, and each embedded symbol point introduces two slope lines crossing at the symbol point, as in Fig. 2(a). The totality of all the slope lines introduced by embedded symbol points is an oblique grid, which divide the generation plane into different positions (Fig. 2(b)).

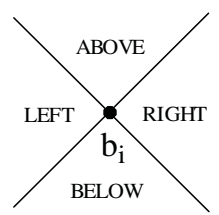

(a)

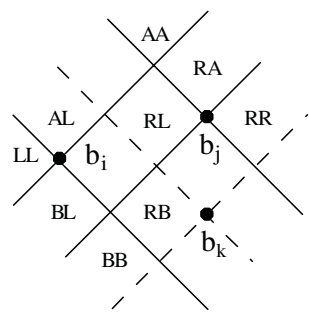

(b)

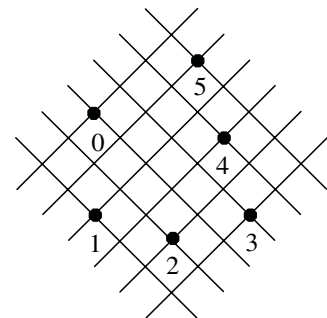

(c)

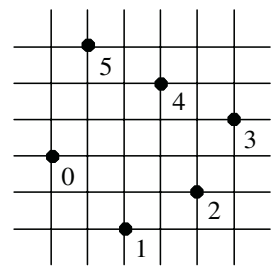

(d)

Fig. 2. The generation plane. (a) divided into 4 positions by $b_{i}$ (b) 9 positions by $b_{i}$ and $b_{j}, 16$ positions after $b_{k}$ embedded (c) oblique grid after 6 symbols embedded (d) after rotated by -45 degree. The SP of these six blocks is $(051423,120345)$

Take an arbitrary order of the $n$ blocks, and then embed them orderly. For blocks $b_{i}$ and $b_{j}, b_{j}$ must be embedded in one of the 4 unique positions according to $b_{i}$, named: LEFT, BELOW, RIGHT and ABOVE (Fig. 2(a)), to satisfy their relation. Blocks must satisfy their relations with all embedded ones by locating at a proper position.

When all blocks are embedded, there will be $2 \mathrm{n}$ lines and $\mathrm{n}^{2}$ intersection points. The $\mathrm{n}$ symbol points locate on $\mathrm{n}$ of $\mathrm{n}^{2}$ intersection points, and no two symbols locate on the same line simultaneously. Fig. 2(c) shows the generation plane after blocks 0 to 5 of Fig. 1 are embedded. As can be easily seen from Fig. 2(d), the sequences of the block names in column (on the vertical lines) and in row (on horizontal lines) are the $\Gamma_{+}$and $\Gamma_{-}$of the Sequence Pair to be generated, respectively.

\footnotetext{
${ }^{1}$ See: http://www.cse.ucsc.edu/research/surf/GSRC/MCNCbench.html
} 


\section{A Generation Algorithm}

Make use of two linked list spx and spy to represent the $\Gamma_{+}$and $\Gamma_{-}$respectively, and generate spx and spy separately by inserting the block indices into the lists. The relations according to their Feasible-HVRS define the orders of two blocks in the lists respectively, and in either list, $b_{i}$ is before $b_{k}$ if $b_{i}$ is before $b_{j}$ and $b_{j}$ is before $b_{k}$. The following program section illustrates the construction of $\mathrm{spx}$.

Construction of list spx in $\mathrm{C}++$ (the block indices are $0,1,2, \ldots, \mathrm{n}-1$ )

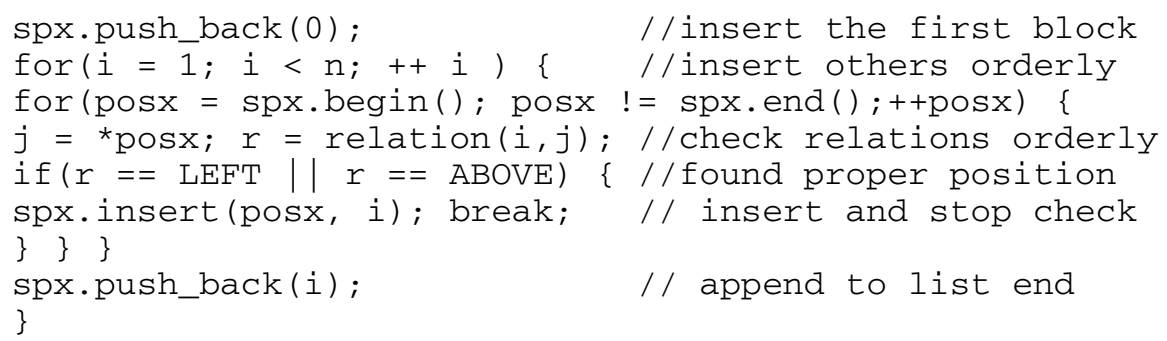

\section{Analytical and Experimental Results}

The relation of every two given blocks has four possibilities. Without loss of generality, the block names to be embedded in sequence are assumed as $1,2,3, \ldots \mathrm{n}$. Before embedding the $k^{\text {th }}$ block, every position introduced by the $k-1$ embedded block symbols is different from others, providing the proper number of unique positions.

Let $p_{k}$ denote the different ways for the $k^{\text {th }}$ block to be embedded, which is evidently $k^{2}$. This generation process comprises the sequence to embed blocks one by one, so the total ways for the embedment of $\mathrm{n}$ blocks can be calculated by multiplying all $p_{k}$, and thus the solution space size of $\mathrm{P}^{*}$-admissible representations is $(\mathrm{n} !)^{2}$.

In general, it needs $n(n-1)=O\left(n^{2}\right)$ time complexity to determine the Feasible-HVRS of $n$ blocks. All the positions in the list can be assumed equiprobable, and thus the average comparison times for inserting the block $b_{k+1}$ into a list with $k$ blocks is:

$$
C_{k+1}=\frac{1}{k+1} \sum_{i=0}^{k} i=\frac{k}{2} \quad(k=0,1,2, \ldots, n-1)
$$

Replacing $k+1$ with $k$, let $C_{k}$ denote the average comparison times for the $k^{\text {th }}$ block, the total average comparison times $C$ to sum up is in Eq. (2). Although its complexity is still $\mathrm{O}\left(\mathrm{n}^{2}\right), C$ in Eq. (2) is only a quarter of the former $\mathrm{n}(\mathrm{n}-1)$ running time.

$$
C=\sum_{k=1}^{n} C_{k}=\sum_{k=1}^{n} \frac{k-1}{2}=\frac{1}{4} n(n-1)
$$

Table 1 shows the running process of the algorithm to generate the spx from the placement in Fig. 1. The count of comparisons is 20, approximating to a quarter of $n(n-1)=9 * 8=72$. If complicated data structures employed, it will be much faster. 
Table 1. Run of the algorithm after block 0 inserted in list spx. A, B, L and R stand for relations between two blocks of Above, Below, Left to and Right to respectively

\begin{tabular}{llllll}
\hline$\#$ & Comparisons & List & \# & Comparisons & List \\
\hline $\mathbf{1}$ & B0 & 01 & $\mathbf{5}$ & R0, R1, A4 & 015423 \\
$\mathbf{2}$ & B0, R1, & 012 & $\mathbf{6}$ & A0 & 6015423 \\
$\mathbf{3}$ & R0, R1, R2 & 0123 & $\mathbf{7}$ & R6, A0 & 67015423 \\
$\mathbf{4}$ & R0, R1, A2 & 01423 & $\mathbf{8}$ & R6, R7, R0, R1, L5 & 670185423 \\
\hline
\end{tabular}

Experiments on all the MCNC benchmarks are run repeatedly in comparison with the generation routine from Parquet [7] on running time. Our results save average $91.5 \%$ time. And the more complex the circuit is, the more time is saved.

\section{Conclusion}

In this paper, we introduce a new method to generate Sequence Pair, and show on the basis of the method that for any $\mathrm{P}^{*}$-admissible placement representation if it takes for granted that every two blocks only have vertical and horizontal relations, the size of its solution space should be as large as $(n !)^{2}$. A quick generation algorithm is also proposed according to the method to reduce the running time. Analytical and experimental results of the algorithm both show its high performance.

\section{References}

1. Murata, H., et al., Rectangle-packing-based module placement. 1995 IEEE/ACM ICCAD. Digest of Technical Papers, (1995):. 472-479.

2. Nakatake, S., et al., Module packing based on the BSG-structure and IC layout applications. IEEE TCAD Of Integrated Circuits And Systems, (1998). 17(6): 519-530.

3. Jai-Ming, L. and C. Yao-Wen, TCG: a transitive closure graph-based representation for non-slicing floorplans. Proceedings of the 38th DAC, (2001): 764-769.

4. Lin, J.M. and Y.W. Chang, TCG-S: Orthogonal coupling of $\mathrm{P}^{*}$-admissible representations for general floorplans, in 39th DAC, Proceedings. (2002). 842-847.

5. Onodera, H., Y. Taniguchi, and K. Tamaru, Branch-and-bound placement for building block layout. 28th ACM/IEEE DAC. Proceedings 1991, (1991): 433-439.

6. Murata, H., et al., A mapping from sequence-pair to rectangular dissection. Proceedings of the ASP-DAC '97. 1997, (1997): 625-633.

7. Adya, S.N. and I.L. Markov, Fixed-outline floorplanning through better local search. Proceedings 2001 IEEE ICCD: VLSI in Computers and Processors. 2001, (2001): 328-334. 